Bull. Korean Math. Soc. 51 (2014), No. 4, pp. 1101-1113

http://dx.doi.org/10.4134/BKMS.2014.51.4.1101

\title{
CONNECTIONS ON REAL PARABOLIC BUNDLES OVER A REAL CURVE
}

\author{
SAnjay Amrutiya
}

\begin{abstract}
We give analogous criterion to admit a real parabolic connection on real parabolic bundles over a real curve. As an application of this criterion, if real curve has a real point, then we proved that a real vector bundle $E$ of rank $r$ and degree $d$ with $\operatorname{gcd}(r, d)=1$ is real indecomposable if and only if it admits a real logarithmic connection singular exactly over one point with residue given as multiplication by $-\frac{d}{r}$. We also give an equivalent condition for real indecomposable vector bundle in the case when real curve has no real points.
\end{abstract}

\section{Introduction}

By a real curve, we mean a pair $\left(X, \sigma_{X}\right)$, where $X$ is a compact Riemann surface and $\sigma_{X}$ is an anti-holomorphic involution on $X$. A real vector bundle over a real curve $\left(X, \sigma_{X}\right)$ is a pair $\left(E, \sigma^{E}\right)$, where $\pi: E \rightarrow X$ is a holomorphic vector bundle and $\sigma^{E}$ is an anti-holomorphic involution on $E$ such that $\pi \circ \sigma^{E}=$ $\sigma_{X} \circ \pi$ and for all $x \in X$, the map $\left.\sigma^{E}\right|_{E(x)}: E(x) \rightarrow E(\sigma(x))$ is $\mathbb{C}$-antilinear:

$$
\sigma^{E}(\lambda \cdot \eta)=\bar{\lambda} \cdot \sigma^{E}(\eta) \text { for all } \lambda \in \mathbb{C} \text { and all } \eta \in E(x),
$$

where $E(x)$ denotes the fibre of $E$ over $x \in X$. Let $\left(X, \sigma_{X}\right)$ be a real curve, and let $\left(E, \sigma^{E}\right)$ be a real vector bundle over $\left(X, \sigma_{X}\right)$. Let $S \subset X$ be a non-empty finite subset of $X$ such that $\sigma_{X}(S)=S$. By a real parabolic structure on $\left(E, \sigma^{E}\right)$ over $S$ we mean for each $x \in S$, a strictly decreasing weighted flag in $E(x)$ which is preserved by $\sigma^{E}$ and the weights over $x$ and $\sigma_{X}(x)$ are same (see Section 4 for the precise definition). In [1], we have established the equivariant description of real parabolic bundles for a suitable ramified covering in the category of real curves.

A theorem due to A. Weil says that a holomorphic vector bundle $E$ over a compact connected Riemann surface admits a holomorphic connection if and only if each direct summand of $E$ is of degree zero (see [2], [5]). In [3] it was proved that parabolic holomorphic vector bundle $E$ admits a parabolic connection if and only if the parabolic degree of every parabolic vector bundle

Received August 26, 2013; Revised December 13, 2013.

2010 Mathematics Subject Classification. Primary 14H60, 14P99; Secondary 14E20.

Key words and phrases. real parabolic bundles, real holomorphic connection, real curve. 
which is a direct summand of $E$ is zero. See [4] for more general statement and different approach to the similar result on parabolic bundles.

In this paper, we prove the analogue of the above two results for real curves. In Section 3, we prove that a real vector bundle $\left(E, \sigma^{E}\right)$ admits a real connection if and only if every real direct summand of $\left(E, \sigma^{E}\right)$ is of degree zero (see Proposition 3.7).

In Section 4, we prove that a real parabolic bundle $\left(W, \sigma^{E}\right)$ with real parabolic structure over $S$ admits a real parabolic connection if and only if every real parabolic direct summand of $\left(E, \sigma^{E}\right)$ is of parabolic degree zero (see Theorem 4.4). As a consequence of Theorem 4.4, we obtained that a real parabolic semistable vector bundles of parabolic degree zero admits a real parabolic connection (see Corollary 4.5).

In Section 5, we prove the following proposition as an application of the main Theorem 4.4. Assume that $\sigma_{X}$ has a fixed point. Let $x_{0} \in X$ be such that $\sigma_{X}\left(x_{0}\right)=x_{0}$.

Proposition 5.1. Let $\left(E, \sigma^{E}\right)$ be a real vector bundle over $X$ of rank $r$ and degree $d$ with $\operatorname{gcd}(r, d)=1$. Then the following are equivalent:

(1) The real vector bundle $\left(E, \sigma^{E}\right)$ is real indecomposable.

(2) There is a real logarithmic connection $D$ on $E$ singular exactly over $x_{0}$ with residue

$$
\operatorname{Res}\left(D, x_{0}\right)=-\frac{d}{r} \mathbf{1}_{E_{x_{0}}} .
$$

If $\sigma_{X}$ has no fixed points, then also we prove the similar result for real indecomposable bundle (see Proposition 5.2).

\section{Preliminaries}

By a real curve, we mean a pair $\left(X, \sigma_{X}\right)$, where $X$ is a compact Riemann surface and $\sigma_{X}$ is an anti-holomorphic involution on $X$. Let $\sigma_{\mathbb{C}}: \mathbb{C} \rightarrow \mathbb{C}$ be the conjugate map $z \mapsto \bar{z}$.

Proposition 2.1. A continuous involution $\sigma: X \rightarrow X$ on a Riemann surface $X$ is an anti-holomorphic involution if and only if for every open subset $U$ of $X$, the map

$$
\tilde{\sigma}=\tilde{\sigma}_{U}: \mathcal{O}_{X}(U) \rightarrow \mathcal{O}_{X}(\sigma(U))
$$

defined by $f \mapsto \sigma_{\mathbb{C}} \circ f \circ \sigma$ is an isomorphism of rings.

Proof. If $\sigma: X \rightarrow X$ is an anti-holomorphic involution, then the map $\tilde{\sigma}_{U}$ defined as above will be an isomorphism. Conversely, suppose that the map

$$
\tilde{\sigma}=\tilde{\sigma}_{U}: \mathcal{O}_{X}(U) \rightarrow \mathcal{O}_{X}(\sigma(U))
$$

defined by $f \mapsto \sigma_{\mathbb{C}} \circ f \circ \sigma$ is an isomorphism. For every pair of charts $\psi_{1}: U_{1} \rightarrow$ $V_{1} \subset \mathbb{C}$ and $\psi_{2}: U_{2} \rightarrow V_{2} \subset \mathbb{C}$ on $X$ with $\sigma\left(U_{1}\right) \subset U_{2}$, the map

$$
\psi_{2} \circ \sigma \circ \psi_{1}^{-1}: V_{1} \rightarrow V_{2}
$$


is anti-holomorphic, since $\sigma_{\mathbb{C}} \circ \psi_{2} \circ \sigma$ is holomorphic. This proves that the map $\sigma: X \rightarrow X$ is an anti-holomorphic involution.

\section{Real vector bundles}

Let $\left(X, \sigma_{X}\right)$ be a real curve. A real holomorphic vector bundle $E \rightarrow X$ is a holomorphic vector bundle, together with an anti-holomorphic involution $\sigma^{E}$ of $E$ making the diagram

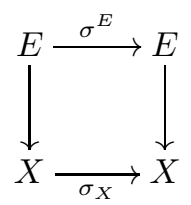

commutative such that for all $x \in X$, the map $\left.\sigma^{E}\right|_{E(x)}: E(x) \rightarrow E(\sigma(x))$ is $\mathbb{C}$-antilinear:

$$
\sigma^{E}(\lambda \cdot \eta)=\bar{\lambda} \cdot \sigma^{E}(\eta) \text { for all } \lambda \in \mathbb{C} \text { and all } \eta \in E(x) .
$$

A homomorphism between two real bundles $\left(E, \sigma^{E}\right)$ and $\left(E^{\prime}, \sigma^{E^{\prime}}\right)$ is a homomorphism

$$
f: E \rightarrow E^{\prime}
$$

of holomorphic vector bundles over $X$ such that $f \circ \sigma^{E}=\sigma^{E^{\prime}} \circ f$.

A holomorphic subbundle $F$ of a real holomorphic vector bundle $E$ is said to be real subbundle of $E$ if $\sigma^{E}(F)=F$.

\section{Real $\mathcal{O}_{X^{-}}-$modules}

Let $(X, \sigma)$ be a real curve, and let $\mathcal{F}$ be an $\mathcal{O}_{X}$-module. We define an $\mathcal{O}_{X^{-}}$ module $\mathcal{F}^{\sigma}$ as follows. For any open subset $U$ of $X, \mathcal{F}^{\sigma}(U)=\mathcal{F}(\sigma(U))$, and for every $f \in \mathcal{O}_{X}(U)$ and $s \in \mathcal{F}^{\sigma}(U), f \cdot s=\tilde{\sigma}_{U}(f) s$. It is easy to check that $\mathcal{F}^{\sigma}$ is an $\mathcal{O}_{X}$-module.

Let $\phi: \mathcal{F} \rightarrow \mathcal{G}$ be a homomorphism of $\mathcal{O}_{X}$-modules. Define $\phi^{\sigma}: \mathcal{F}^{\sigma} \rightarrow \mathcal{G}^{\sigma}$ as follows: For every open subset $U$ of $X$,

$$
\phi_{U}^{\sigma}: \mathcal{F}^{\sigma}(U) \rightarrow \mathcal{G}^{\sigma}(U), \phi_{U}^{\sigma}=\phi_{\sigma(U)} .
$$

If $f \in \mathcal{O}_{X}(U)$, and $s \in \mathcal{F}^{\sigma}(U)$, then

$$
\phi_{U}^{\sigma}(f \cdot s)=\phi_{\sigma(U)}\left(\sigma_{U}(f)(s)\right)=\sigma_{U}(f) \phi_{\sigma(U)}(s),
$$

since $\phi_{\sigma(U)}$ is an $\mathcal{O}_{X}(\sigma(U))$-linear. Therefore, $\phi^{\sigma}(f \cdot s)=f \cdot \phi^{\sigma}(s)$. It follows that $\phi^{\sigma}$ is a homomorphism of $\mathcal{O}_{X}$-modules.

Definition 2.2. A real structure on an $\mathcal{O}_{X}$-module $\mathcal{F}$ is an $\mathcal{O}_{X}$-module homomorphism $\sigma^{\mathcal{F}}: \mathcal{F} \rightarrow \mathcal{F}^{\sigma}$ such that $\left(\sigma^{\mathcal{F}}\right)^{\sigma} \circ \sigma^{\mathcal{F}}=\mathbf{1}_{\mathcal{F}}$. By a real $\mathcal{O}_{X}-$ module, we mean a pair $\left(\mathcal{F}, \sigma^{\mathcal{F}}\right)$, where $\mathcal{F}$ is an $\mathcal{O}_{X}$-module and $\sigma^{\mathcal{F}}$ is a real structure on an $\mathcal{O}_{X}$-module $\mathcal{F}$.

Let $\left(\mathcal{F}, \sigma^{\mathcal{F}}\right)$ and $\left(\mathcal{G}, \sigma^{\mathcal{G}}\right)$ be two real $\mathcal{O}_{X}$-modules. A morphism $\phi: \mathcal{F} \rightarrow \mathcal{G}$ of $\mathcal{O}_{X}-$ modules is said to be a morphism of real $\mathcal{O}_{X}$ modules if $\sigma^{\mathcal{G}} \circ \phi=\phi^{\sigma} \circ \sigma^{\mathcal{F}}$. 
Remark 2.3. Let $E$ be a real holomorphic vector bundle over a real curve $X$. Then the corresponding locally free $\mathcal{O}_{X}$-module $\mathcal{E}$ is a real $\mathcal{O}_{X}$-module. Conversely, if $\mathcal{E}$ is a locally free real $\mathcal{O}_{X}$-module, then the corresponding holomorphic vector bundle is a real holomorphic vector bundle.

\section{Connections on real vector bundles}

Let $\left(E, \sigma^{E}\right)$ be a real vector bundle over $\left(X, \sigma_{X}\right)$. A holomorphic connection $D$ on $E$ is a $\mathbb{C}$-linear sheaf morphism

$$
D: E \rightarrow E \otimes K_{X}
$$

which satisfies the Leibniz identity, $D(f s)=f D(s)+d f \otimes s$, where $f$ is any locally defined holomorphic function on $X$ and $s$ any local holomorphic section of $E$.

Define $D^{\sigma}: E^{\sigma} \rightarrow \Omega_{X}^{1}(E)^{\sigma}$ by $D_{U}^{\sigma}(s)=D_{\sigma(U)}(s)$ for $U$ open subset of $X$, and $s \in E^{\sigma}(U)$. Then $D^{\sigma}$ is also a holomorphic connection on $E^{\sigma}$.

Definition 3.1. A holomorphic connection $D$ is called a real holomorphic connection (or just real connection) on $\left(E, \sigma^{E}\right)$ if $\sigma^{E} \circ D=D^{\sigma} \circ \sigma^{E}$.

Proposition 3.2. Let $\left(E, \sigma^{E}\right)$ be a real indecomposable vector bundle over $\left(X, \sigma_{X}\right)$. Let $\phi$ be a real endomorphism of $\left(E, \sigma^{E}\right)$. Then one of the following holds:

- $\phi=\lambda \mathbf{1}_{E}+\psi$, where $\lambda \in \mathbb{R}$ and $\psi$ is a nilpotent real endomorphism of $\left(E, \sigma^{E}\right)$,

- $E \cong F \oplus F^{\sigma}$, where $\sigma^{E}(F)=F^{\sigma}$ and in this case, $\phi=(\lambda, \bar{\lambda}) \mathbf{1}_{E}+\psi$, where $\lambda \in \mathbb{C}$ and $\psi$ is a nilpotent real endomorphism of $\left(E, \sigma^{E}\right)$.

Proof. First note that the characteristic polynomial of $\phi(x) \in \operatorname{End}(E(x))$ does not depend on $x$, as $X$ is compact and connected. Consider the decomposition of $E$ obtained from generalized eigenspace decomposition for $\phi$. Since $\phi$ is a real endomorphism and $\left(E, \sigma^{E}\right)$ is real indecomposable, we either have only one eigenvalue $\lambda \in \mathbb{R}$ or have two eigenvalues $\lambda$ and $\bar{\lambda}$, where $\lambda \in \mathbb{C}$. In the case where there is only one eigenvalue, consider $\psi=\phi-\lambda \mathbf{1}_{E}$. Then $\psi$ is a real endomorphism. By Fitting decomposition

$$
E=\operatorname{Ker}\left(\psi^{n}\right) \oplus \operatorname{Im}\left(\psi^{n}\right)
$$

for sufficiently large integer $n$. Since $\psi$ is real, the subsheaves $\operatorname{Ker}\left(\psi^{n}\right)$ and $\operatorname{Im}\left(\psi^{n}\right)$ are torsion-free real $\mathcal{O}_{X}$-submodules of $\left(E, \sigma^{E}\right)$, and hence real subbundles of $\left(E, \sigma^{E}\right)$. Since $\left(E, \sigma^{E}\right)$ is real indecomposable, we must have either $\operatorname{Ker}\left(\psi^{n}\right)=0$ or $\operatorname{Im}\left(\psi^{n}\right)=0$. Let $v \in E(x)$ be an eigenvector of $\phi(x): E(x) \rightarrow$ $E(x)$ for the eigenvalue $\lambda$. Then, $\psi(x) v=0$ and $v \neq 0$, so $\psi$ can not be an isomorphism, and hence we must have $\operatorname{Im}\left(\psi^{n}\right)=0$, that is, $\psi$ is nilpotent.

In the case where $\phi$ has two eigenvalues $\lambda$ and $\bar{\lambda}$, then we have $E=F \oplus F^{\sigma}$, where $F$ correspond to $\lambda$ and $F^{\sigma}$ correspond to $\bar{\lambda}$. Note that $\sigma^{E}(F)=F^{\sigma}$. 
Consider the endomorphism $\psi:=\phi-(\lambda, \bar{\lambda}) \mathbf{1}_{E}$. Then $\psi$ is a real endomorphism, since

$$
\begin{aligned}
\sigma^{E} \circ(\lambda, \bar{\lambda}) \mathbf{1}_{E}\left(v_{1}, v_{2}\right) & =\sigma^{E}\left(\lambda v_{1}, \bar{\lambda} v_{2}\right) \\
& =\left(\lambda \sigma^{E}\left(v_{2}\right), \bar{\lambda} \sigma^{E}\left(v_{1}\right)\right) \\
& =(\lambda, \bar{\lambda}) \mathbf{1}_{E}\left(\sigma^{E}\left(v_{2}\right), \sigma^{E}\left(v_{1}\right)\right) \\
& =(\lambda, \bar{\lambda}) \mathbf{1}_{E} \circ \sigma^{E}\left(v_{1}, v_{2}\right) .
\end{aligned}
$$

Now the assertion follows by the similar argument as given above.

Lemma 3.3. Let $\left(E, \sigma^{E}\right)$ be a real vector bundle over $\left(X, \sigma_{X}\right)$. If $E$ admits a holomorphic connection, then it admits a real connection.

Proof. Let $D_{1}: E \rightarrow \Omega_{X}^{1}(E)$ be a holomorphic connection on $E$. Let

$$
D_{1}^{\prime}:=\sigma^{E} \circ D_{1}^{\sigma} \circ \sigma^{E}: E \rightarrow \Omega_{X}^{1}(E) .
$$

Consider the average

$$
D:=\frac{D_{1}+D_{1}^{\prime}}{2}
$$

Then

$$
\begin{aligned}
D(f s) & =1 / 2\left(D_{1}(f s)+D_{1}^{\prime}(f s)\right) \\
& =1 / 2\left(f D_{1}(s)+d f \otimes s+f D_{1}^{\prime}(s)+d f \otimes s\right) \\
& =1 / 2\left(D_{1}+D_{1}^{\prime}\right)(f s)+d f \otimes s \\
& =D(f s)+d f \otimes s .
\end{aligned}
$$

Moreover,

$$
\begin{aligned}
\sigma^{E} \circ D & =1 / 2\left(\sigma^{E} \circ D_{1}+D_{1}^{\sigma} \circ \sigma^{E}\right) \\
& =1 / 2\left(D_{1}{ }^{\sigma} \circ \sigma^{E}+D_{1}^{\sigma} \circ \sigma^{E}\right) \\
& =D \circ \sigma^{E} .
\end{aligned}
$$

Therefore, $D$ is a real connection on $\left(E, \sigma^{E}\right)$.

Lemma 3.4. Let $E_{1}$ and $E_{2}$ be two real vector bundles over $X$. The direct sum $E_{1} \oplus E_{2}$ admits a real connection if and only if both $E_{1}$ and $E_{2}$ admit a real connection.

Proof. If $D_{1}$ and $D_{2}$ are real connections on $E_{1}$ and $E_{2}$ respectively, then $D_{1} \oplus D_{2}$ is a real connection on $E_{1} \oplus E_{2}$.

Conversely, if $D$ is a real connection on the real vector bundle $E_{1} \oplus E_{2}$, then the differential operator

$$
\left(q_{E_{i}} \otimes \mathbf{1}_{K_{X}}\right) \circ D \circ \iota_{E_{i}}: E_{i} \rightarrow E_{i} \otimes K_{X},
$$

$i=1,2$, is a real connection on the real vector bundle $E_{i}$, where $\iota_{E_{i}}: E_{i} \hookrightarrow$ $E_{1} \oplus E_{2}$ is the inclusion morphism, and

$$
q_{E_{i}}: E_{1} \oplus E_{2} \rightarrow E_{i}
$$

the natural projection. This completes the proof. 
Remark 3.5. Let $\left(E, \sigma^{E}\right)$ be a real vector bundle over $X$. We have Atiyah exact sequence

$$
0 \rightarrow \operatorname{End}(E) \rightarrow \operatorname{At}(E) \rightarrow T X \rightarrow 0
$$

Recall that a holomorphic connection on $E$ is a holomorphic splitting of the exact sequence (3.1). There is a nondegenerate $\mathbb{C}$-bilinear pairing

$$
\langle\bullet, \bullet\rangle: H^{1}\left(X, K_{X} \otimes \operatorname{End}(E)\right) \times H^{0}(X, \operatorname{End}(E)) \rightarrow \mathbb{C}
$$

given by

$$
\langle a, \phi\rangle=\int_{X} \operatorname{Tr}(\alpha \phi),
$$

where $\alpha \in A^{1,1}(\operatorname{End}(E))(X)$ is a Dolbeault representative of $a$. If $E$ is a real vector bundle, then there is an induced real structure on $H^{1}\left(X, K_{X} \otimes \operatorname{End}(E)\right)$ and $H^{0}(X, \operatorname{End}(E))$ which we will denote by $\sigma$ to ease the notations. Then by the definition of pairing it is clear that

$$
\langle\sigma(a), \phi\rangle=\langle a, \sigma(\phi)\rangle
$$

for $a \in H^{1}\left(X, K_{X} \otimes \operatorname{End}(E)\right)$ and $\phi \in H^{0}(X, \operatorname{End}(E))$.

Let

$$
\operatorname{at}(E) \in H^{1}\left(X, K_{X} \otimes \operatorname{End}(E)\right)=H^{0}(X, \operatorname{End}(E))^{*}
$$

be the Atiyah class representing (3.1). Let $\phi \in H^{0}(X, \operatorname{End}(E))$. Then

$$
\operatorname{at}(E)(\phi)= \begin{cases}2 \pi \sqrt{-1} \operatorname{deg}(E) & \text { if } \phi=\mathbf{1}_{E} ; \\ 0 & \text { if } \phi \text { is nilpotent. }\end{cases}
$$

For more details see [2].

Lemma 3.6. If $\left(E, \sigma^{E}\right)$ is a real indecomposable vector bundle over $\left(X, \sigma_{X}\right)$ of degree zero. Then $\left(E, \sigma^{E}\right)$ admits a real connection.

Proof. Let $\left(E, \sigma^{E}\right)$ be a real indecomposable vector bundle over $\left(X, \sigma_{X}\right)$ of degree zero. To show that $\left(E, \sigma^{E}\right)$ admits a real connection, it is enough to show that $\operatorname{at}(E)=0$. Let $\phi \in H^{0}(X, \operatorname{End}(E))$. Then the endomorphism $\phi+\sigma(\phi)$ of $E$ is real endomorphism. Since $E$ is real indecomposable, by Proposition 3.2 we have

- either $\phi+\sigma(\phi)=\lambda \mathbf{1}_{E}+\psi$, where $\lambda \in \mathbb{R}$ and $\psi$ is a nilpotent real endomorphism of $\left(E, \sigma^{E}\right)$,

- or $E \cong F \oplus F^{\sigma}$, where $\sigma^{E}(F)=F^{\sigma}$ and in this case, $\phi+\sigma(\phi)=$ $(\lambda, \bar{\lambda}) \mathbf{1}_{E}+\psi$, where $\lambda \in \mathbb{C}$ and $\psi$ is a nilpotent real endomorphism of $\left(E, \sigma^{E}\right)$.

In both the cases, from (3.5) it follows that

$$
\operatorname{at}(E)(\phi+\sigma(\phi))=0 \text {. }
$$

Recall that

$$
\operatorname{at}(E)(\phi)=-\int_{X} \operatorname{Tr}(R \phi),
$$


where $R$ is the curvature of the unique $C^{\infty}$ unitary connection on $E$ (with respect to a fix $C^{\infty}$ Hermitian metric $h$ in $E$ ) that is compatible with the holomorphic structure of $E$. Since $E$ is real vector bundle, $R$ is real form and hence from (3.4) it follows that $\operatorname{at}(E)(\phi)=\operatorname{at}(E)(\sigma(\phi))$. From (3.6) we can conclude that $\operatorname{at}(E)(\phi)=0$.

Proposition 3.7. Let $\left(E, \sigma^{E}\right)$ be a real vector bundle over $\left(X, \sigma_{X}\right)$. Then $E$ admits a real connection if and only if every real direct summand of $\left(E, \sigma^{E}\right)$ is of degree zero.

Proof. This follows by combining Lemma 3.3, Lemma 3.4 and Lemma 3.6.

\subsection{Connection on real equivariant vector bundles}

Let $\left(Y, \sigma_{Y}\right)$ be a real curve. Let $G$ be a finite group acting holomorphically and effectively on $Y$ with the property that $\sigma_{Y}(g y)=g^{-1} \sigma_{Y}(y)$ for all $g \in G$.

Definition 3.8. A $G$-equivariant real vector bundle on $\left(Y, \sigma_{Y}\right)$ consists of the following data: a real holomorphic vector bundle $\left(W, \sigma^{W}\right)$ on $\left(Y, \sigma_{Y}\right)$, and a lift of the natural action of $G$ on $Y$ to $W$ such that

(a) the bundle projection $\pi: W \rightarrow Y$ is $G$-equivariant;

(b) if $y \in Y$ and $g \in G$, the map $W(y) \rightarrow W(g \cdot y)$, given by $v \mapsto g \cdot v$ is a linear isomorphism.

(c) the following diagram

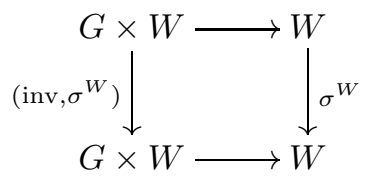

commutes, where inv : $G \rightarrow G$ is an inverse map $g \mapsto g^{-1}$.

We say that a real connection $D$ on $W$ is a real $G$-connection if it is preserved by the $G$-action. Since $G$ is finite, by averaging, we see that if $W$ admits a real connection, then it admits a real $G$-connection. The following is immediate:

Proposition 3.9. If $W$ is indecomposable as real $G$-equivariant bundle of degree zero, then $W$ admits a real $G$-connection.

Proof. Let $\phi$ be an endomorphism of $W$. Then

$$
\phi^{\prime}=\sum_{g \in G} g(\phi+\sigma(\phi))
$$

is a real $G$-equivariant endomorphism of $W$. Since $W$ is indecomposable as real $G$-equivariant bundle of degree zero, using the similar arguments as in Proposition 3.2 and Lemma 3.6 we have at $(W)\left(\phi^{\prime}\right)=0$. From the proof of $[3$, Lemma 2.2], it follows that the Atiyah class at $(W) \in H^{1}\left(Y, K_{Y} \otimes \operatorname{End}(\mathrm{W})\right)$ is fixed by the action of $G$. Therefore,

$$
|G| \operatorname{at}(W)(\phi+\sigma(\phi))=0,
$$


where $|G|$ denotes the order of the group $G$. Since at $(W)(\phi)=\operatorname{at}(W)(\sigma(\phi))$, we have $\operatorname{at}(W)(\phi)=0$.

Theorem 3.10. A real $G$-equivariant bundle $W$ admits a real $G$-connection if and only if every real $G$-equivariant direct summand of $W$ is of degree zero.

\section{Real parabolic connection on real parabolic bundles}

Definition 4.1. Let $\left(E, \sigma^{E}\right)$ be a real vector bundle over a real curve $\left(X, \sigma_{X}\right)$. Let $S$ be a finite subset of $X$ such that $\sigma_{X}(S)=S$.

By real quasi-parabolic structure on $\left(E, \sigma^{E}\right)$ over $S$, we mean for each $x \in S$, there is a strictly decreasing flag

$$
E(x)=F^{1} E(x) \supset F^{2} E(x) \supset \cdots \supset F^{k_{x}} E(x) \supset F^{k_{x}+1} E(x)=0
$$

of linear subspaces in $E(x)$ satisfying the following property:

(RP1) $\sigma^{E}$ preserve the flags, i.e., $\sigma_{x}^{E}\left(F^{i} E(x)\right)=F^{i} E\left(\sigma_{X}(x)\right)$.

We define

$$
r_{j}=\operatorname{dim}\left(F^{j} E(x)\right)-\operatorname{dim}\left(F^{j+1} E(x)\right) .
$$

The integer $k_{x}$ is called the length of the flag and the sequence $\left(r_{1}, \ldots, r_{k_{x}}\right)$ is called the type of the flag.

A real parabolic structure on $\left(E, \sigma^{E}\right)$ over $S$ is a real quasi-parabolic structure on $\left(E, \sigma^{E}\right)$ over $S$ as above, together with a sequence of real numbers $0 \leq \alpha_{1}^{x}<\cdots<\alpha_{k_{x}}^{x}<1$, which are called weights corresponding to the subspaces $\left(F^{1} E(x), F^{2} E(x), \ldots, F^{k_{x}} E(x)\right)$, with the following property:

(RP2) the weights over $x$ and $\sigma_{X}(x)$ are same.

We set

$$
d_{x} E=\sum_{j=1}^{k_{x}} r_{j} \alpha_{j},
$$

where $r_{j}=\operatorname{dim}\left(F^{j} E(x)\right)-\operatorname{dim}\left(F^{j+1} E(x)\right)$.

The parabolic degree, denoted by $\operatorname{pdeg}(E)$, is defined by

$$
\operatorname{pdeg}(E)=\operatorname{deg}(E)+\sum_{x \in S} d_{x} E,
$$

where $\operatorname{deg}(E)$ denotes the topological degree of $E$, and we define the parabolic slope by

$$
\mathrm{p} \mu(E)=\frac{\operatorname{pdeg}(E)}{\operatorname{rank}(E)} .
$$

We recall the definition of real parabolic semistable bundles over a real curve (see [1]). A real parabolic bundle $\left(E, \sigma^{E}\right)$ is called real parabolic semistable if for every real parabolic subbundle $F$ of $E$, we have

$$
\mathrm{p} \mu(F) \leq \mathrm{p} \mu(E) .
$$


Definition 4.2. A real logarithmic connection on $\left(E, \sigma^{E}\right)$ singular over $S$ is a first order differential operator

$$
D: E \rightarrow \Omega_{X}^{1}(E)(S):=E \otimes K_{X} \otimes \mathcal{O}_{X}(S)
$$

which satisfy the following:

(1) $\sigma^{\Omega_{X}^{1}(E)(S)} \circ D=D^{\sigma} \circ \sigma^{E}$, and

(2) $D(f s)=f D(s)+s \otimes d f$, where $f$ is a locally defined function and $s$ is a locally defined section of $E$.

Recall that there is a well define endomorphism of $E(x)$ for each $x \in S$, so called, residue of $D$ at $x$, denoted by $\operatorname{Res}(D, x)$. Because of the condition (1) in Definition $4.2, \operatorname{Res}(D, x)$ is a real endomorphism for each $x \in S$, that is, the following diagram

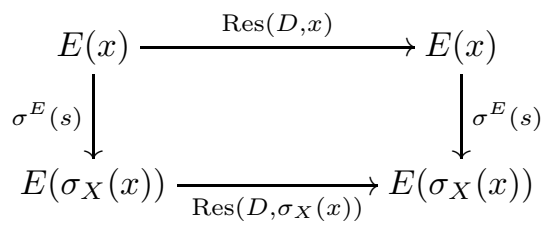

commutes for all $x \in S$.

Let $\left(E, \sigma^{E}\right)$ be a real parabolic vector bundle on $\left(X, \sigma_{X}\right)$ with real parabolic structure over $S$.

Definition 4.3. A real parabolic connection on $\left(E, \sigma^{E}\right)$ is a real logarithmic connection $D$ on $\left(E, \sigma^{E}\right)$, singular over $S$, satisfying the following conditions:

(RC) for any $x \in S, \operatorname{Res}(D, x)\left(F^{i} E(x)\right) \subset F^{i} E(x)$ and

$$
\left.\operatorname{Res}(D, x)\right|_{F^{i} E(x) / F^{i+1} E(x)}=\alpha_{i}^{x} \cdot \mathbf{1}_{F^{i} E(x) / F^{i+1} E(x)}
$$

for all $i \in\left\{1,2, \ldots, k_{x}\right\}$, where $k_{x}$ as in (4.1).

Theorem 4.4. A real parabolic bundle $\left(E, \sigma^{E}\right)$ admits a real parabolic connection if and only if every real parabolic direct summand of $\left(E, \sigma^{E}\right)$ is of parabolic degree zero.

Proof. Let $\left(E, \sigma^{E}\right)$ be a real parabolic bundle on $\left(X, \sigma_{X}\right)$ with real parabolic structure over $S$. Let $N$ be a positive integer such that all the weights of $\left(E, \sigma^{E}\right)$ are integral multiple of $1 / N$. By [1, Lemma 4.1], there exists an $N$ fold cyclic ramified covering $p:\left(Y, \sigma_{Y}\right) \rightarrow\left(X, \sigma_{X}\right)$ which is ramified over each point of $S$. Let $\Gamma$ be a Galois group of the covering $p$. By $[1$, Proposition $5.1]$, there exists a real $\Gamma$-equivariant vector bundle $W$ over $\left(Y, \sigma_{Y}\right)$ such that $p_{*}^{\Gamma} W$ (with induced real parabolic structure) is isomorphic to $\left(E, \sigma^{E}\right)$ as a real parabolic bundle on $\left(X, \sigma_{X}\right)$ with real parabolic structure over $S$. Note that there is a one-to-one correspondence between real subbundles of $\left(E, \sigma^{E}\right)$ and real $\Gamma$-invariant subbundles of $W[1]$. Recall that

$$
|\Gamma| \cdot \operatorname{pdeg}(E)=\operatorname{deg} W,
$$


where $|\Gamma|$ denotes the order of the group $\Gamma$. Suppose that every real parabolic direct summand of $\left(E, \sigma^{E}\right)$ is of parabolic degree zero. Since real subbundles of $\left(E, \sigma^{E}\right)$ are in one-to-one correspondence with the real $\Gamma$-invariant subbundles of $W$, using (4.4) it follows that every real direct summand of the real $\Gamma$ equivariant vector bundle $W$ is of degree zero. By Theorem 3.10, it follows that $W$ admits a real $\Gamma$-connection, say $D^{\prime}: W \rightarrow W \otimes K_{Y}$. Since $K_{Y} \sim p^{*} K_{X}+R_{p}$, where $R_{p}$ is the ramification divisor of $p$, we can consider the composition

$$
W \rightarrow W \otimes K_{Y} \rightarrow W \otimes p^{*} K_{X} \otimes \mathcal{O}_{Y}\left(R_{p}\right) .
$$

Note that $\mathcal{O}_{Y}\left(R_{p}\right)=p^{*} \mathcal{O}_{X}(D)$, where $D=\sum_{x \in S} s$. Hence, from (4.5), we obtain $W \rightarrow W \otimes p^{*}\left(K_{X} \otimes \mathcal{O}_{X}(D)\right)$. By taking direct image and using projection formula, we get the differential operator

$$
\tilde{D}: p_{*} W \rightarrow p_{*} W \otimes K_{X}(D)
$$

satisfying the Leibniz identity. Since $D^{\prime}$ is real $\Gamma$-connection, $\tilde{D}$ is a real $\log$ arithmic connection on $p_{*} W$ and commutes with the natural action of $\Gamma$ on $p_{*} W$. Therefore, $\tilde{D}$ induces a logarithmic connection on the invariant direct image $\left(p_{*} W\right)^{\Gamma}$. Now recall that the parabolic structure on $\left(p_{*} W\right)^{\Gamma}$ (see [1, Proposition 5.1]) is given as follows: For $x \in S$, let $y$ be a ramified point of $p$ over $x$. For the isotropy generator $\xi$, the distinct eigen-values of the operator $\xi: W(y) \rightarrow W(y)$ will be $\omega^{k_{1}}, \omega^{k_{2}}, \ldots, \omega^{k_{r_{x}}}$ with multiplicity $n_{1}, \ldots, n_{r_{x}}$, where $0 \leq k_{1}<\cdots<k_{r_{x}}<N$ and $\omega=\exp 2 \pi \sqrt{-1} / N$. By reindexing, we can write $0 \leq k_{1}^{\prime} \leq k_{2}^{\prime} \leq \cdots \leq k_{r}^{\prime}$ according to their multiplicities, where $r=\operatorname{rk}(W)$. Let $V_{i}$ be the $\omega^{k_{i}}$-eigenspace in $W(y)$ of the isotropy $\xi$. Then the real parabolic structure on $\left(p_{*} W\right)^{\Gamma}$ over $S$ is given by

$$
\left(p_{*} W\right)^{\Gamma}(s) \supset F_{y}^{1} \supset F_{y}^{2} \supset \cdots \supset F_{y}^{r_{x}} \supset F_{y}^{r_{x}+1}=0,
$$

where $F_{y}^{i}=V_{i} \oplus \cdots \oplus V_{r_{x}}$ with associated weight $a_{i}=k_{i} / N$ for $i=1, \ldots, r_{x}$.

Consider the weights $0 \leq \alpha_{x}^{1} \leq \alpha_{x}^{2} \leq \cdots \leq \alpha_{x}^{r}$ according to their multiplicities, where $\alpha_{x}^{i}=k_{i}^{\prime} / N$. Consider the local model of $p$. Let

$$
\mathbb{D}=\{z \in \mathbb{C}|| z \mid<1\}
$$

be the open unit disk, and let

$$
p: \mathbb{D} \rightarrow \mathbb{D}
$$

be the holomorphic map defined by $z \mapsto z^{N}$. Consider the trivial vector bundle $W_{\mathbb{D}}:=\mathbb{D} \times \mathbb{C}^{r}$ of $\operatorname{rank} r=\operatorname{rk}(W)$. The action of the isotropy generator of $\Gamma$ on $W_{\mathbb{D}}$ is given by

where $\Delta_{0}=\operatorname{diag}\left(\omega^{k_{1}^{\prime}}, \ldots, \omega^{k_{r}^{\prime}}\right)$.

$$
(z, v) \mapsto\left(\omega z, \Delta_{0}(v)\right),
$$

From this local description and the induced real parabolic structure on $\left(p_{*} W\right)^{\Gamma}$, it follows that the residue $\operatorname{Res}(D, x)$ preserves the quasi-parabolic structure and it acts on $F^{i} E(x) / F^{i+1} E(x)$ as multiplication by $k_{i}^{x} / N$ for any $x \in S$. 
Conversely, assume that a real parabolic bundle $\left(E, \sigma^{E}\right)$ admits a real parabolic connection, say $D^{\prime}$. The pullback of the real connection $D^{\prime}$ is indeed a real $\Gamma$-invariant connection on restriction of $W$ to $Y \backslash T$, where $T=p^{-1}(S)$. This induced connection on $\left.W\right|_{Y \backslash T}$ extends to $W$ over $Y$. Recall that the real parabolic direct summands of $E$ are in one-to-one correspondence with real $\Gamma$ equivariant direct summands of $W$. By Theorem 3.10 and (4.4), it follows that every real parabolic direct summand of $\left(E, \sigma^{E}\right)$ is of parabolic degree zero.

Corollary 4.5. Every real parabolic semistable vector bundle of parabolic degree zero admits a real parabolic connection.

Proof. If $\left(E, \sigma^{E}\right)$ is a real parabolic semistable vector bundles of parabolic degree zero, then the parabolic degree of every real parabolic vector bundle which is direct summand of $\left(E, \sigma^{E}\right)$ (as real parabolic bundle) is zero. Therefore, the corollary follows from Theorem 4.4 .

\section{Real indecomposable bundles and real logarithmic connections}

Let $\left(X, \sigma_{X}\right)$ be a real curve. Assume that $\sigma_{X}$ has a fixed point. Let $x_{0} \in X$ be such that $\sigma_{X}\left(x_{0}\right)=x_{0}$.

Proposition 5.1. Let $\left(E, \sigma^{E}\right)$ be a real vector bundle over $X$ of rank $r$ and degree $d$ with $\operatorname{gcd}(r, d)=1$. Then the following are equivalent:

(1) The real vector bundle $\left(E, \sigma^{E}\right)$ is real indecomposable.

(2) There is a real logarithmic connection $D$ on $E$ singular exactly over $x_{0}$ with residue $\operatorname{Res}\left(D, x_{0}\right)=-\frac{d}{r} \mathbf{1}_{E\left(x_{0}\right)}$.

Proof. Assume that $\left(E, \sigma^{E}\right)$ is real indecomposable. Since $r$ and $d$ are coprime, we can write $d=r k+d_{0},-r<d_{0}<0$. Let $E^{\prime}:=E \otimes \mathcal{O}_{X}\left(-k x_{0}\right)$. Since $\sigma\left(x_{0}\right)=x_{0}$, the vector bundle $E^{\prime}$ is real vector bundle. Clearly, the real vector bundle $\left(E^{\prime}, \sigma^{E^{\prime}}\right)$ is real indecomposable if and only if $\left(E, \sigma^{E}\right)$ is real indecomposable. The de Rham differential $f \mapsto d f$ defines a real logarithmic connection $D_{0}$ on the real line bundle $\mathcal{O}_{X}\left(-k x_{0}\right)$ singular exactly over $x_{0}$. The residue of $D_{0}$ at $x_{0}$ is $k$.

The real vector bundle $\left(E^{\prime}, \sigma^{E^{\prime}}\right)$ is equipped with the following real parabolic structure over $x_{0}$. The real quasi-parabolic structure is the trivial one, i.e., $E^{\prime}\left(x_{0}\right) \supset 0$, and the parabolic weight of $E^{\prime}\left(x_{0}\right)$ is $\frac{-d_{0}}{r}$. Note that $0<\frac{-d_{0}}{r}<1$. We have

$$
\operatorname{pdeg}\left(E^{\prime}\right)=\operatorname{deg}\left(E^{\prime}\right)+d_{x_{0}} E^{\prime}=d_{0}-r \cdot \frac{d_{0}}{r}=0 .
$$

Using Theorem 4.4 it follows that there is a real logarithmic connection $D^{\prime}$ on $E^{\prime}$ singular exactly over $x_{0}$ with residue $\operatorname{Res}\left(D^{\prime}, x_{0}\right)=-\frac{d_{0}}{r} \mathbf{1}_{E^{\prime}\left(x_{0}\right)}$. This real logarithmic connection $D^{\prime}$ on $E^{\prime}$ with residue $-\frac{d_{0}}{r} \mathbf{1}_{E^{\prime}\left(x_{0}\right)}$ and the de Rham logarithmic connection on $\mathcal{O}_{X}\left(k x_{0}\right)$ together induce a real logarithmic connection on $E$ singular exactly over $x_{0}$ with residue $-\frac{d}{r} \mathbf{1}_{E\left(x_{0}\right)}$. 
Conversely, assume that there is a real logarithmic connection $D$ on $E$ singular exactly over $x_{0}$ with residue $\operatorname{Res}\left(D, x_{0}\right)=-\frac{d}{r} \mathbf{1}_{E\left(x_{0}\right)}$. Then the real parabolic bundle $\left(E^{\prime}, \sigma^{E^{\prime}}\right)$ (defined above) admits a real parabolic connection. By Theorem 4.4 for each real direct summand of $E^{\prime}$, the real parabolic subbundle $F$ with induced real parabolic structure is of parabolic degree zero. Suppose $\left(E^{\prime}, \sigma^{E}\right)=\left(E_{1}, \sigma^{E_{1}}\right) \oplus\left(E_{2}, \sigma^{E_{2}}\right)$. With the induced real parabolic structure on $\left(E_{i}, \sigma^{E_{i}}\right)$, we have

$$
\operatorname{pdeg}\left(E_{i}\right)=d_{i}-r_{i} \cdot \frac{d_{0}}{r}=0,
$$

where $r_{i}=\operatorname{rank}\left(E_{i}\right) \neq 0$ and $d_{i}=\operatorname{deg}\left(E_{i}\right)$ with $d_{1}+d_{2}=d_{0}$. This implies that

$$
\frac{d_{i}}{r_{i}}=\frac{d_{0}}{r}
$$

From this it follows that $r$ and $d$ are not coprime, which is a contradiction. Therefore, the real vector bundle $E^{\prime}$ is real indecomposable and hence $E$ is real indecomposable.

We now assume that $\sigma_{X}$ has no fixed point. Let $S$ be a non-empty finite subset of $X$ such that $\sigma_{X}(S)=S$. Then the cardinality of $S$ is even, say $n$. Let us write $S=\left\{x_{1}, x_{2}, \ldots, x_{n-1}, x_{n}\right\}$, where $\sigma_{X}\left(x_{i}\right)=x_{i+1}$ whenever $i$ is odd.

Proposition 5.2. Let $\left(E, \sigma^{E}\right)$ be a real vector bundle over $X$ of rank $r$ and degree $d$ with $\operatorname{gcd}(r, d)=1$. Then the following are equivalent:

(1) The real vector bundle $\left(E, \sigma^{E}\right)$ is real indecomposable.

(2) There is a real logarithmic connection $D$ on $E$ singular exactly over each point of $S$ with residue at each point of $S$ as follows:

(a) $\operatorname{Res}\left(D, x_{1}\right)=\frac{-d-(2 n-1)}{n r} \mathbf{1}_{E\left(x_{1}\right)}$,

(b) $\operatorname{Res}\left(D, x_{2 j}\right)=\frac{-d+(n+1)}{n r} \mathbf{1}_{E\left(x_{2 j}\right)}, j=1, \ldots, \frac{n}{2}$,

(c) $\operatorname{Res}\left(D, x_{2 j-1}\right)=\frac{-d-(n-1)}{n r} \mathbf{1}_{E\left(x_{2 j-1}\right)}, j=2, \ldots, \frac{n}{2}$.

Proof. Consider the divisor

$$
\Delta=-2 k x_{1}+k x_{2}+\sum_{i=3}^{n}(-1)^{i-1} k x_{i},
$$

where $k$ is as in the proof of Proposition 5.1. Set $E^{\prime}:=E \otimes \mathcal{O}_{X}(\Delta)$. Note that $E^{\prime}$ is a real vector bundle equipped with the following real parabolic structure over $S$ : The real quasi-parabolic structure is trivial and the weights over each point of $S$ are same given by $-\frac{d_{0}}{n r}$, where $d_{0}=d-r k$ (see Definition 4.1 ). We have

$$
\operatorname{pdeg}\left(E^{\prime}\right)=\operatorname{deg}\left(E^{\prime}\right)+\sum_{x \in S} d_{x} E^{\prime}=d_{0}-r \cdot \frac{n d_{0}}{n r}=0 .
$$

The rest of the proof is similar to Proposition 5.1 with simple computation. 
Remark 5.3. In the second statement of Proposition 5.2, one can interchange the residue at $x_{2 j}$ and $x_{2 j-1}$. In that case one has to change the definition of the divisor $\Delta$ used in the proof of Proposition 5.2 in order to get the proof.

Acknowledgement. The author would like to thank the anonymous referee for helpful comments and suggestions. The present form of Proposition 5.2 is made after the referee's suggestion.

\section{References}

[1] S. Amrutiya, Real parabolic bundles over a real curve, Proc. Indian Acad. Sci. (Math. Sci.). 124 (2014), 17-30.

[2] M. F. Atiyah, Complex analytic connections in fibre bundles, Trans. Amer. Math. Soc. 85 (1957), 181-207.

[3] I. Biswas, A criterion for the existence of a flat connection on a parabolic vector bundle, Adv. Geom. 2 (2002), no. 3, 231-241.

[4] W. Crawley-Boevey, Indecomposable parabolic bundles and the existence of matrices in prescribed conjugacy class closures with product equal to the identity, Publ. Math. Inst. Hautes Études. Sci. 100 (2004), 171-207.

[5] A. Weil, Généralisation des fonctions abéliennes, J. Math. Pures Appl. 17 (1938), 47-87.

Institute of Mathematical Sciences

CIT CAMPUS

Taramani, Chennai 600 113, India

E-mail address: amrutiya@imsc.res.in 